\title{
Síntese e caracterização de materiais mesoporosos modificados com níquel para a captura de $\mathrm{CO}_{2}$
}

\author{
(Synthesis and characterization of mesoporous materials \\ modified by nickel for capture of $\mathrm{CO}_{2}$ )
}

\author{
A. R. Nascimento ${ }^{1}$, G. P. Figueredo ${ }^{1}$, G. Rodrigues ${ }^{1}$, M. A. F. Melo ${ }^{1}$, M. J. B. Souza ${ }^{2}$, D. M. A. Melo ${ }^{1}$ \\ ${ }^{1}$ Laboratório de Catálise do NUPPRAR, Universidade Federal do Rio Grande do Norte - UFRN, \\ Campus Universitário Lagoa Nova, C.P. 1524, Natal, RN, Brasil 59078-970 \\ ${ }^{2}$ Universidade Federal de Sergipe - UFS, Cidade Universitária Prof. José Aloísio de Campos, \\ Av. Marechal Rondon, s/n, Jardim Rosa Elze, S. Cristóvão, SE, Brasil 49100-000 \\ ale3ufs@yahoo.com.br
}

\begin{abstract}
Resumo
Materiais mesoporosos tipo SBA-15 e MCM-48 foram sintetizados com sucesso pelo método hidrotérmico e impregnados com níquel nas proporções de 5-20 peso\% visando à captura de $\mathrm{CO}_{2}$. Os materiais obtidos foram caracterizados por meio de análise por difração de raios X, adsorção de $\mathrm{N}_{2}$ e microscopia eletrônica de varredura. Testes de captura de $\mathrm{CO}_{2}$ foram conduzidos em pressões entre 100 e $4000 \mathrm{KPa}$ à temperatura constante de $298 \mathrm{~K}$ e revelaram que as capacidades de adsorção variam significativamente com as propriedades texturais dos materiais com diferentes teores de níquel, atingindo valores máximos de 14,89 e $9,97 \mathrm{mmol} \mathrm{g}^{-1}$ para MCM-48 e SBA-15, respectivamente.

Palavras-chave: SBA-15, MCM-48, materiais mesoporosos, níquel, captura de $\mathrm{CO}_{2}$.
\end{abstract}

\section{Abstract}

SBA-15 and MCM-48 mesoporous materials were successfully synthesized by the hydrothermal method and impregnated with nickel in proportions of 5-20 wt.\% aiming to capture $\mathrm{CO}_{2}$. The materials were characterized by X-ray diffraction analysis, $\mathrm{N}_{2}$ adsorption and scanning electron microscopy. $\mathrm{CO}_{2}$ capture tests were conducted at pressures between 100 and $4000 \mathrm{KPa}$ at a constant temperature of $298 \mathrm{~K}$ and showed that the adsorption capacities vary significantly with the textural properties of these materials with different contents of nickel, reaching maximum values of 14.89 and $9.97 \mathrm{mmol} \mathrm{g}^{-1}$ for MCM-48 and SBA-15, respectively.

Keywords: SBA-15, MCM-48, mesoporous materials, nickel, $\mathrm{CO}_{2}$ capture.

\section{INTRODUÇÃO}

A quantidade de $\mathrm{CO}_{2}$ liberada na atmosfera pode ser reduzida através da utilização de fontes alternativas de energia, tais como energia hidrelétrica, eólica, solar, nuclear, entre outras. Cada uma delas tem suas limitações e ainda haverá grande dependência de combustíveis fósseis para o transporte e produção de energia por algum tempo $[1,2]$. Durante a queima desses combustíveis há uma elevada produção de $\mathrm{CO}_{2}$, que é liberado diretamente na atmosfera. Portanto, a captura e estocagem de $\mathrm{CO}_{2}$ será uma importante contribuição para a redução da emissão desse gás poluente.

A captura de $\mathrm{CO}_{2}$ pode ser realizada diretamente nas principais fontes de emissão, como as usinas que geram eletricidade; nas indústrias de produção de aço, ferro e cimento que geram $\mathrm{CO}_{2}$ como subprodutos; e na exploração do gás natural antes de sua utilização como combustível [3]. Os principais combustíveis fósseis utilizados em usinas de energia são gás e carvão, que são queimados na presença de ar produzindo o $\mathrm{CO}_{2}$, através da ligação do oxigênio do ar ao carbono do combustível. Para que o $\mathrm{CO}_{2}$ seja armazenado de forma eficiente, se faz necessário a sua separação de outros gases de escape. Nesse caso, a técnica mais usada atualmente para captura do $\mathrm{CO}_{2}$ emprega soluções alcalinas que podem solubilizar ou seqüestrar o $\mathrm{CO}_{2}$, a exemplo das aminas. Elas absorvem $\mathrm{CO}_{2}$ através de interações intermoleculares devido ao caráter ácido-base desses constituintes, particularmente quando estão sob alta pressão e baixas temperaturas. Esse processo é chamado de lavagem e a solução resultante é então aquecida e a pressão é reduzida, liberando $\mathrm{CO}_{2}$ concentrado [4]. No entanto, o uso de aminas apresenta desvantagens por ser dispendiosa e causar problemas de corrosão [5]. Por conseguinte, a adsorção gás-sólido vem sendo utilizada como uma técnica de separação promissora para evitar os problemas com o uso da amina, citados anteriormente. Nesse caso, o processo de adsorção ocorre devido à existência de forças atrativas não compensadas na superfície do adsorvente. Dependendo da 
natureza e magnitude das forças envolvidas, poderá haver adsorção física, causada por forças de interação moleculares fracas ou adsorção química, envolvendo o rearranjo dos elétrons do gás que interage com o sólido, formando uma ligação química forte $[6,7]$.

Adsorventes sólidos são materiais que têm boa capacidade de adsorção, são de fácil regeneração, além de apresentar considerável estabilidade química e mecânica durante longo período de ciclo do processo industrial. Diversos materiais estão atualmente em estudo para o processo de captura de $\mathrm{CO}_{2}$ a exemplo dos óxidos metálicos e óxidos metálicos mistos, zeólitas, materiais carbonáceos, estruturas metalorgânicas (MOFs), organosílica e superfícies de sílica modificadas [1, 8]. Estes adsorventes diferem uns dos outros com base na sua estrutura química, composição e tipo de sítios de adsorção [9]. A família de peneiras moleculares de sílica mesoporosa apresenta considerável interesse para aplicaçõe sem áreas como separação, catálise e adsorção [10]. Os materiais mesoporosos MCM-48 e SBA-15 apresentam alta área específica, além de elevada estabilidade térmica, hidrotérmica e mecânica. O MCM-48 (Fig. 1a) possui uma célula unitária de simetria cúbica, com um arranjo de canais intergerminados que não se interseccionam. A abertura de poro tridimensional desse material aumenta o número de interações entre adsorbatos e sítios ativos do adsorvente, resultando em uma maior atividade adsortiva e resistência ao bloqueamento de poros [11, 12]. A SBA-15 (Fig. 1b), constituída por um arranjo de canais hexagonais uniformes, vem se tornando um material promissor em diversos processos, por apresentar maior sistema e volume de poros em comparação ao MCM48, em torno de 300 a $500 \AA ̊$ e até $2,5 \mathrm{~cm}^{3} \mathrm{~g}^{-1}$, respectivamente,
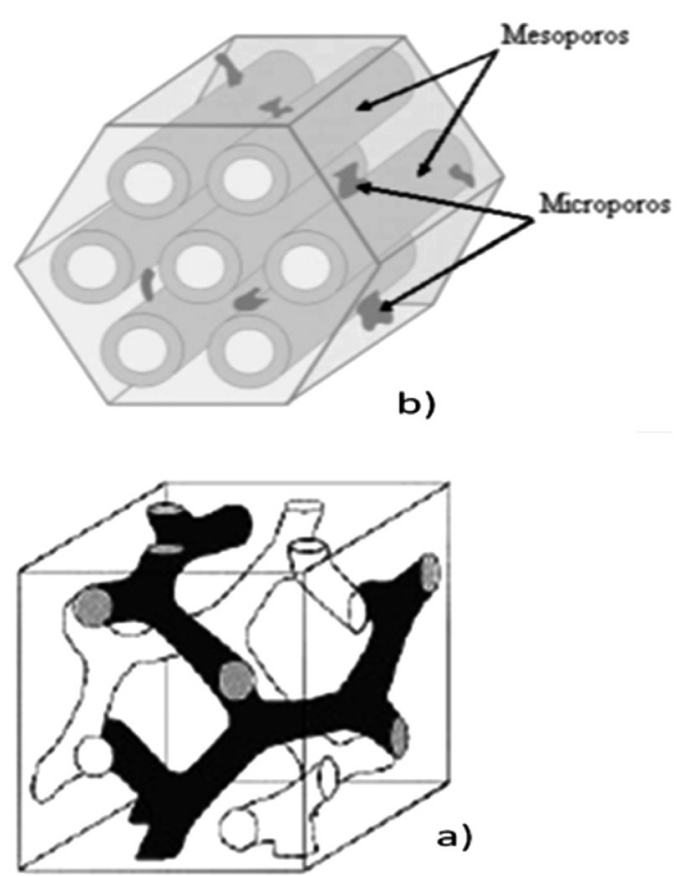

Figura 1: Estrutura mesoporosa do MCM-48 (a) e SBA-15 (b) [13, 14].

[Figure 1: Mesoporous structure of MCM-48 (a) and SBA-15 (b) $[13,14]$. além de espessas paredes dos poros ( 30 a $60 \AA$ ).

A parede dos canais dos materiais mesoporosos é constituída essencialmente por $\mathrm{SiO}_{2}$ amorfo, onde a superfície exibe caráter neutro, apresentando poucos sítios ácidos. O dióxido de carbono é um ácido de Lewis, que aceita elétrons a partir de bases de Lewis (doadores de elétrons) [15]. Nesse sentido, modificações com aminas têm sido propostas no sentido de obter sólidos com melhor propriedade ácido-base. Essas aminas são volumosas e apresentam dificuldade de difusão para o interior dos poros dos materiais mesoporosos [16], reduzindo sua capacidade de adsorção. Assim, partículas de óxidos metálicos apontam como promissores nesses processos de modificação, uma vez que pode melhorar a dispersão nos poros dos materiais. Nesse cenário vem ganhando destaque o óxido de níquel, já amplamente empregado em reações de reforma a seco de metano [17]. Nessa transformação, a etapa determinante é a adsorção do $\mathrm{CO}_{2}$ sobre o níquel metálico depositado sobre o suporte poroso. De modo geral, quanto melhores forem às propriedades texturais do suporte, maior será a capacidade de dispersão da fase ativa e, conseqüentemente, maior a capacidade de adsorção e atividade catalítica. Apesar de suas propriedades texturais diferenciadas, os materiais mesoporosos MCM-48 e SBA-15 não têm sido estudados em processos de modificação com níquel para adsorção de $\mathrm{CO}_{2}$, sendo a potencial aplicação dos óxidos de $\mathrm{Ni}$ em reações de reforma de metano. Na literatura são encontrados trabalhos que apontam resultados animadores da aplicação de materiais mesoporosos modificados com níquel em reações de hidrogenação e benzilação de benzeno $[18,19]$; na reação de etileno/propeno [20], como também no armazenamento de hidrogênio [21]. A capacidade de adsorção é fortemente dependente da área específica e diâmetro de poros dos adsorventes, e, na presença de níquel, o diâmetro e volume de poros foram fundamentais na capacidade de armazenamento de hidrogênio [21]. Portanto, com o objetivo de associar as excelentes propriedades texturais dos materiais mesoporosos e as propriedades de atividade do óxido de níquel em reações de reforma, esse trabalho foi desenvolvido visando estudar o comportamento de adsorção de $\mathrm{CO}_{2}$ em materiais tipo MCM48 e SBA-15 modificados com diferentes teores de níquel para avaliação destes materiais em futuras reações de reforma de combustíveis, como o metano.

Os materiais mesoporosos reportados nesse trabalho têm diâmetros de poro maiores do que o diâmetro cinético do dióxido de carbono [16] e apresentam diferentes arquiteturas e estruturas dos canais porosos, o que torna esse trabalho estratégico na avaliação de diferentes estruturas no processo de impregnação do níquel e na captura de $\mathrm{CO}_{2}$.

\section{MATERIAIS E MÉTODOS}

\section{Sintese hidrotérmica do MCM-48}

A preparação dos adsorventes mesoporosos tipo MCM48 foi realizada através do método hidrotérmico [22] com as devidas adaptações. Foram utilizados TEOS (Sigma Aldrich) 
como fonte de silício, $\mathrm{NaOH}$ (Vetec) como fonte de sódio, CTMABr (Vetec) como direcionador da estrutura e água destilada. As composições químicas molares dos géis de síntese foram obtidas com base na seguinte formula geral: $0,25 \mathrm{Na}_{2} \mathrm{O}-1 \mathrm{SiO}_{2}-0,55 \mathrm{CTMABr}-100 \mathrm{H}_{2} \mathrm{O}$. O procedimento geral de síntese consistiu no preparo de uma solução geral, sem controle de $\mathrm{pH}$,contendo o direcionador da estrutura e hidróxido de sódio na quantidade de água requerida para a síntese. A solução foi submetida à agitação na temperatura de $50{ }^{\circ} \mathrm{C}$ até solubilização do direcionador. Em seguida a fonte de silício (TEOS) foi adicionada lentamente e a solução permaneceu em agitação à temperatura de $50{ }^{\circ} \mathrm{C}$ durante 40 min, sendo observada a formação de um gel branco. O material obtido foi transferido para uma autoclave de teflon encamisado em aço inox e conduzida à estufa mantida a $150{ }^{\circ} \mathrm{C}$ por $24 \mathrm{~h}$. O material obtido foi seco a $100{ }^{\circ} \mathrm{C}$ e calcinado em ar sintético a $550^{\circ} \mathrm{C}$ por $2 \mathrm{~h}$.

\section{Sintese hidrotérmica da SBA-15}

A preparação dos adsorventes mesoporosos tipo SBA-15 foi feita pelo método hidrotérmico, seguindo procedimento reportado [12], com as devidas adaptações. Foram utilizados TEOS (Sigma Aldrich) como fonte de silício, $\mathrm{HCl}$ (Vetec) como diluente, P123 (Sigma Aldrich) como direcionador da estrutura, e água destilada. As composições químicas molares dos géis de síntese foram obtidas com base na seguinte formula geral: $2,8 \mathrm{HCl}-1 \mathrm{SiO}_{2}-0,017 \mathrm{P} 123-180 \mathrm{H}_{2} \mathrm{O}$. O procedimento geral de síntese consistiu no preparo de uma solução geral, sem controle de $\mathrm{pH}$, contendo o copolímero tribloco P123, $\mathrm{HCl}$ concentrado e água destilada. A solução foi submetida a agitação constante a $45^{\circ} \mathrm{C}$, até solubilização do copolímero. Em seguida, foi adicionada lentamente a fonte de silício (TEOS) e a solução permaneceu em agitação por $24 \mathrm{~h} \mathrm{a} 45^{\circ} \mathrm{C}$. O material obtido foi colocado em autoclave de teflon encamisado em aço inox e conduzido à estufa mantida a $100^{\circ} \mathrm{C}$ por $24 \mathrm{~h}$. O material resultante foi seco em temperatura ambiente e calcinado em ar sintético a $500^{\circ} \mathrm{C}$ por $2 \mathrm{~h}$.

\section{Impregnação das amostras}

As amostras de MCM-48 e SBA-15 foram impregnadas com níquel nas proporções $5 \%, 10 \%$ e $20 \%(\mathrm{~m} / \mathrm{m})$, usando uma solução de nitrato de níquel hexahidratado dissolvido em álcool isopropílico, por impregnação pós-síntese através do processo de contato. Os cálculos para as concentrações de níquel desejadas foram realizadas em função da massa das amostras utilizadas. Cada adsorvente, juntamente com o nitrato de níquel dissolvido em álcool, foi submetido à agitação por $2 \mathrm{~h}$ à temperatura ambiente, e, após esse tempo, os materiais foram secos em estufa a $60{ }^{\circ} \mathrm{C}$ por $2 \mathrm{~h}$. Os adsorventes obtidos foram calcinados a $450^{\circ} \mathrm{C}$ por $2 \mathrm{~h}$, sob o fluxo de $100 \mathrm{~mL} \mathrm{~min}^{-1}$ de ar sintético.

\section{Caracterização das amostras}

A análise de DRX pelo método do pó foi feita nas amostras MCM-48, 5Ni/MCM-48, 10Ni/MCM-48，20Ni/ MCM-48 e SBA-15, 5Ni/SBA-15, 10Ni/SBA-15, 20Ni/SBA15 , nas faixas angulares $2^{\circ}-80^{\circ}$ e $0,5^{\circ}-80^{\circ}$, respectivamente, com o objetivo de verificar se houve a formação das estruturas cúbica e hexagonal mesoporosas, como também identificar a presença do níquel na estrutura dos materiais analisados.

A determinação das áreas superficiais dos adsorventes obtidos foi feita correlacionando os dados das isotermas de adsorção de nitrogênio a $77 \mathrm{~K}$ pelo modelo BET [23] na faixa de $\mathrm{P} / \mathrm{P}_{0}$ 0,05-0,35. As distribuições de diâmetros de poros dos materiais mesoporosos foram obtidas correlacionando os valores de volume adsorvido em função da pressão relativa $\left(\mathrm{P} / \mathrm{P}_{0}\right)$ nos algoritmos propostos por BJH [24] em uma faixa de poros 10-1000 A.

As micrografias foram obtidas por microscopia eletrônica de varredura nas amostras MCM-48, 5Ni/MCM-48, 10Ni/ MCM-48, 20Ni/MCM-48 e SBA-15, 5Ni/SBA-15, 10Ni/ SBA-15, 20Ni/SBA-15, com o objetivo de observar a morfologia dos materiais adsorventes sintetizados.

\section{Teste de adsorção}

Os testes de adsorção foram realizados pelo método gravimétrico, esquematizado na Fig. 2, em um microadsorvedor Rubotherm, constituído de uma cesta onde é colocada $0,3 \mathrm{~g}$ de adsorvente e inserido em um recipiente resistente à pressão em até $5000 \mathrm{KPa}$. A medida de adsorção alcança o fim quando atinge o equilíbrio, ou seja, sem variação na massa da amostra. Os resultados da medida de adsorção foram expressos por meio de gráficos da relação entre a massa adsorvida e a massa de adsorvente

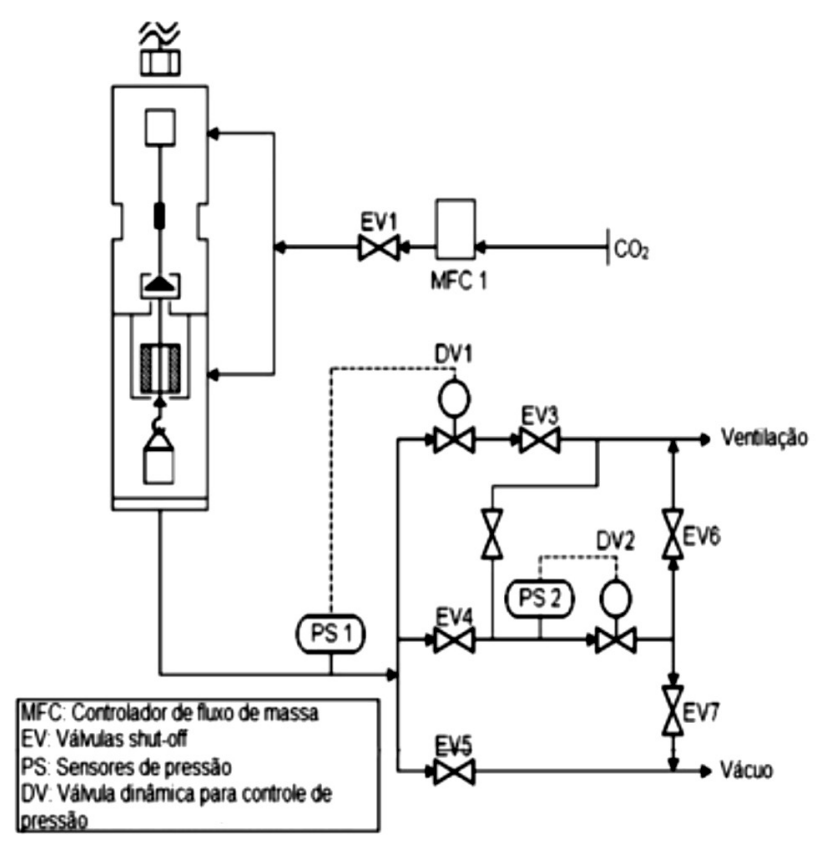

Figura 2: Diagrama esquemático da balança utilizada para de captura de $\mathrm{CO}_{2}$.

[Figure 2: Schematic diagram of the system used to capture of $\mathrm{CO}_{2}$.] 
em função da pressão (isoterma). Os parâmetros utilizados para a realização das análises foram: temperatura (298 K); pressão absoluta (100-4000 KPa); temperatura de ativação da amostra (398 K); pressão de ativação da amostra (vácuo); tempo de ativação $(2 \mathrm{~h})$; adsorbato $\left(\mathrm{CO}_{2}\right)$.

\section{RESULTADOS E DISCUSSÃO}

\section{Caracterização dos materiais MCM-48 e SBA-15}

Os difratogramas de raios X para as amostras MCM-48, 5Ni/MCM-48, 10Ni/MCM-48, 20Ni/MCM-48 e SBA-15, $5 \mathrm{Ni} / \mathrm{SBA}-15,10 \mathrm{Ni} / \mathrm{SBA}-15,20 \mathrm{Ni} / \mathrm{SBA}-15$ são apresentados nas Figs. 3-6. Os materiais apresentaram os picos típicos característicos do material $[12,22]$, indicando que as estruturas cúbica (Fig. 3) e hexagonal (Fig. 5) mesoporosas foram mantidas ordenadas mesmo após a impregnação (Figs. 4 e 6). Após calcinação, o nitrato de níquel foi transformado em óxido de níquel e sua presença foi identificada pelo arquivo JCPDS 01-073-1523, pelos picos (20) em 37,16, 43,26 e 62,79 . A presença da fase $\mathrm{NiO}$ é um indicativo de que parte do óxido permaneceu na parte externa do suporte [25].

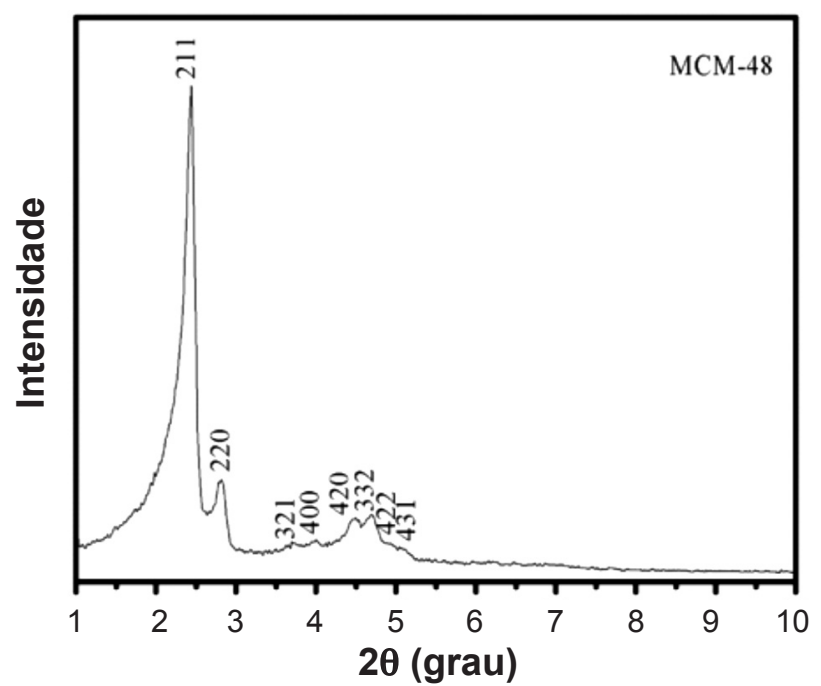

Figura 3: Difratograma de raios X da amostra MCM-48.

[Figure 3: X-ray diffraction pattern of sample MCM-48.]

As Figs. 7 e 8 apresentam as isotermas de adsorção e a distribuição de tamanho de poros das amostras MCM-48, 5Ni/MCM-48, 10Ni/MCM-48, 20Ni/MCM-48 e SBA-15, 5Ni/SBA-15, 10Ni/SBA-15, 20Ni/SBA-15, respectivamente. Em todos os casos foram obtidas isotermas do tipo IV, segundo a classificação da IUPAC, características de materiais mesoporosos. A Fig. 7 mostra que o MCM-48 apresenta histerese tipo H2. Segundo a IUPAC, este tipo de histerese indica que o MCM-48 possui poros com gargalo estreito e corpo largo. $\mathrm{O}$ estreitamento nas histereses em amostras mesoporosas pode ser atribuído a partículas com tamanho de poros em torno de $40 \AA \AA$ (4 nm) ao longo dos canais [26], como pode ser visto na distribuição do tamanho de poros para o MCM-48. A Fig. 8 mostra que a SBA 15 apresentou histerese tipo $\mathrm{H} 1$, indicando que o material possui uma estrutura com canais cilíndricos [12]. A impregnação do SBA-15 proporcionou um aumento na simetria das histereses, sugerindo que houve a formação de uma camada de $\mathrm{NiO}$ no interior dos mesoporos.

Para o MCM-48, o aumento do teor de impregnação reduz a quantidade de poros menores, favorecendo o surgimento dos poros grandes, responsáveis, nesse caso, pelo maior percentual do volume de poros. Por sua vez, na SBA-15, a impregnação com níquel favorece a redução da quantidade de poros grandes, revelando, dessa forma, poros menores, os quais são os principais responsáveis pelo volume de poros referentes a esses materiais. Esse comportamento diferente pode estar relacionado com a diferença na arquitetura dos canais dos poros desses materiais.

As propriedades texturais dos materiais investigados

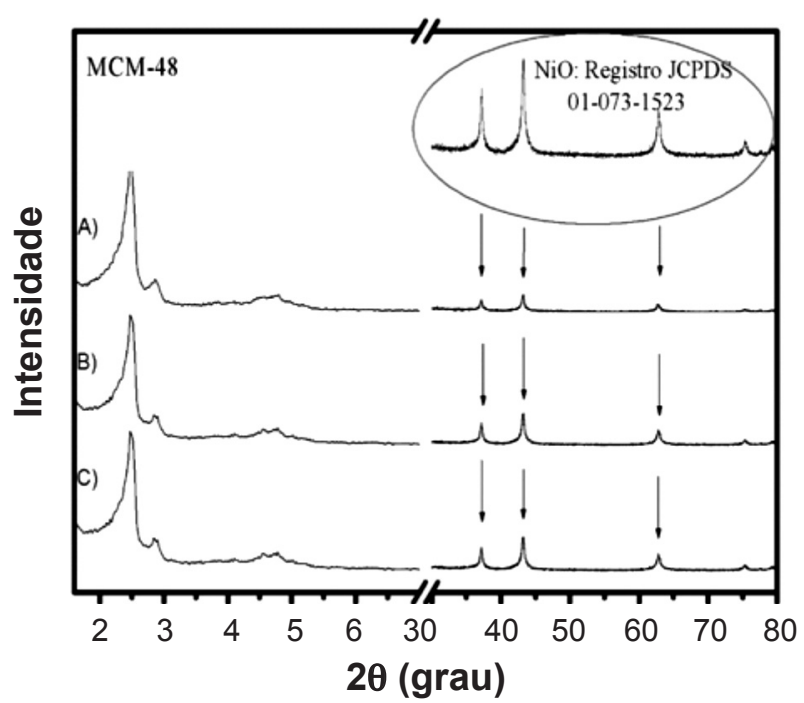

Figura 4: Difratogramas de raios X das amostras (a) 5Ni/MCM-48, (b) $10 \mathrm{Ni} / \mathrm{MCM}-48$ e (c) $20 \mathrm{Ni} / \mathrm{MCM}-48$.

[Figure 4: X-ray diffraction patterns of samples (a) 5Ni/MCM-48, (b) $10 \mathrm{Ni} / \mathrm{MCM}-48$ and (c) $20 \mathrm{Ni} / \mathrm{MCM}-48$.

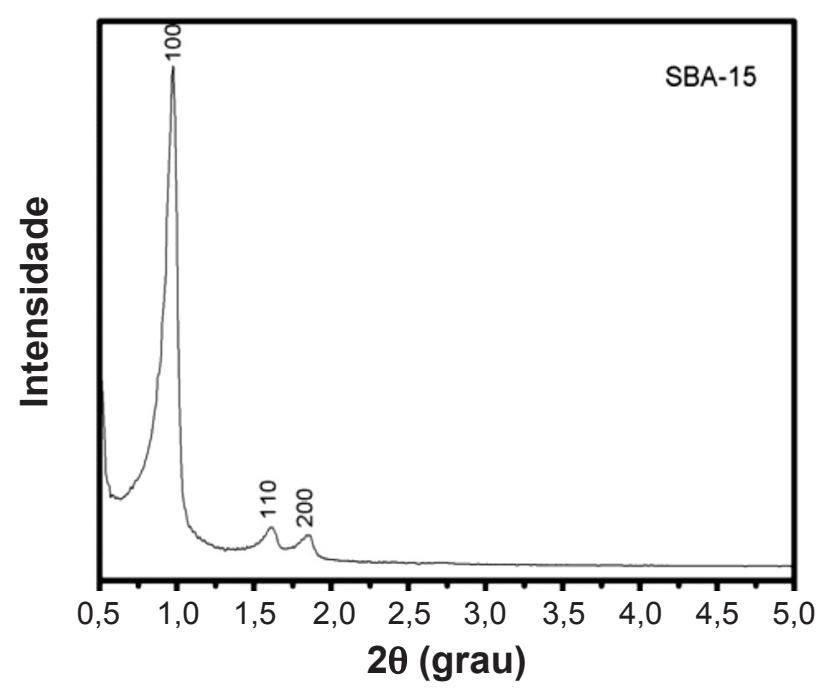

Figura 5: Difratograma de raios X da amostra SBA-15. [Figure 5: X-ray diffraction pattern of sample SBA-15.] 


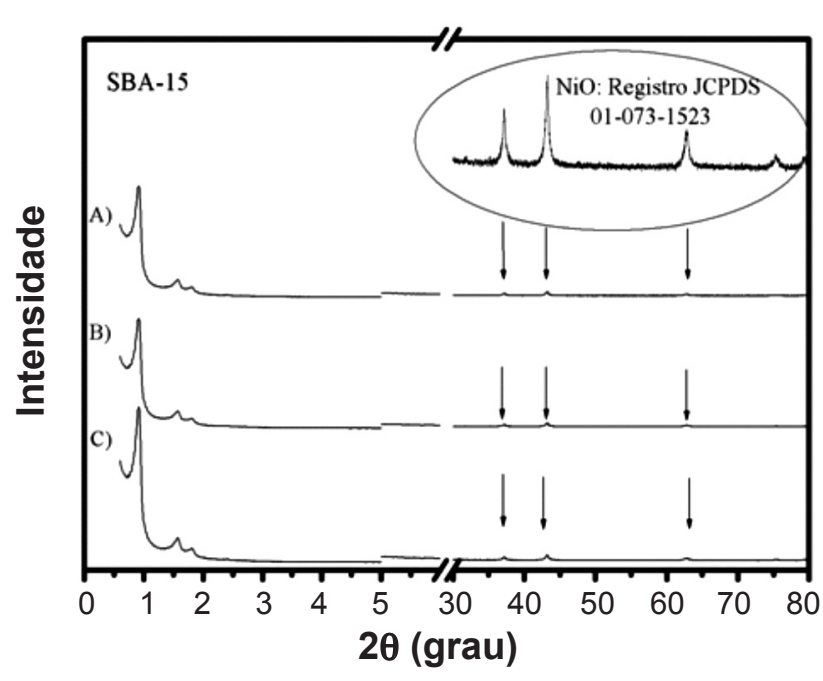

Figura 6: Difratogramas de raios $\mathrm{X}$ das amostras (a) $5 \mathrm{Ni} / \mathrm{SBA}-15$, (b) $10 \mathrm{Ni} / \mathrm{SBA}-15$ e (c) $20 \mathrm{Ni} / \mathrm{SBA}-15$.

[Figure 6: X-ray diffraction patterns of samples (a) $5 \mathrm{Ni} / \mathrm{SBA}-15$, (b) $10 \mathrm{Ni} / \mathrm{SBA}-15$ and (c) $20 \mathrm{Ni} / \mathrm{SBA}-15$.]

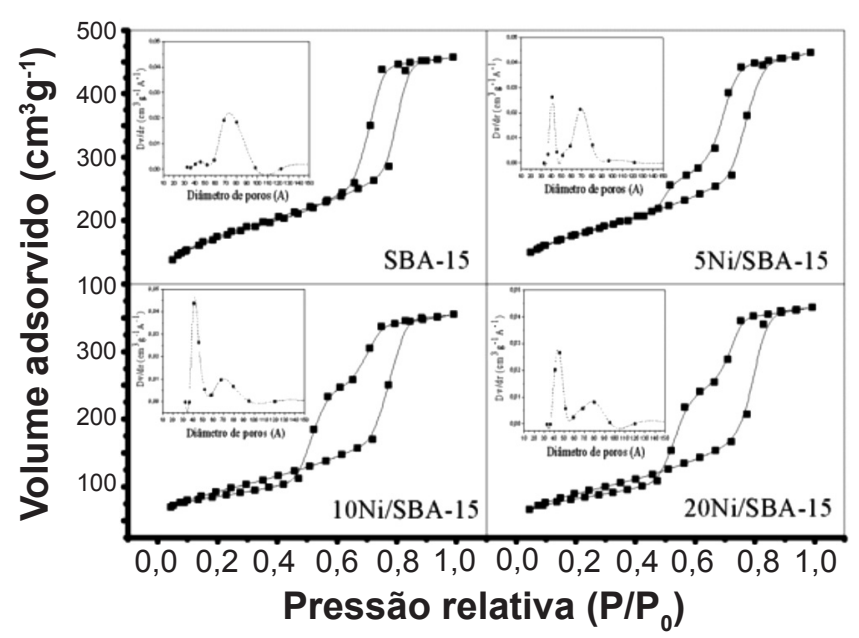

Figura7: Isotermas de adsorção-dessorção de $\mathrm{N}_{2}$ a $77 \mathrm{~K}$ e distribuição de tamanho de poros das amostras MCM-48, 5Ni/MCM-48, 10Ni/MCM-48 e 20Ni/MCM-48.

[Figure 7: $\mathrm{N}_{2}$ adsorption-desorption isotherms at $77 \mathrm{~K}$ and pore size distribution of the samples MCM-48, 5Ni/MCM-48, $10 \mathrm{Ni} / \mathrm{MCM}-48$ and $20 \mathrm{Ni} / \mathrm{MCM}-48$.]

estão indicadas na Tabela I. As faixas de volume de poros e de áreas específicas obtidas para o MCM-48 foram de $0,05 \mathrm{a}$ $0,1 \mathrm{~cm}^{3} \mathrm{~g}^{-1}$ e de 798 a $924 \mathrm{~m}^{2} \mathrm{~g}^{-1}$, respectivamente. A SBA-15 apresentou volumes de poros na faixa de 0,48 a $0,64 \mathrm{~cm}^{3} \mathrm{~g}^{-1}$ e áreas específicas totais na faixa de 248 a $587 \mathrm{~m}^{2} \mathrm{~g}^{-1}$. Para as amostras de SBA-15 impregnadas observa-se uma relação direta entre o aumento do teor de níquel (5, 10 e 20 peso\%) e o aumento da espessura da parede com conseqüente diminuição da área específica. Isso pode estar relacionado ao óxido de níquel depositado na superfície dos poros do material, causando o bloqueio local dos canais porosos, dificultando a difusão do $\mathrm{N}_{2}$ até os sítios ativos presentes no interior dos poros. Essa dificuldade de acesso de gás

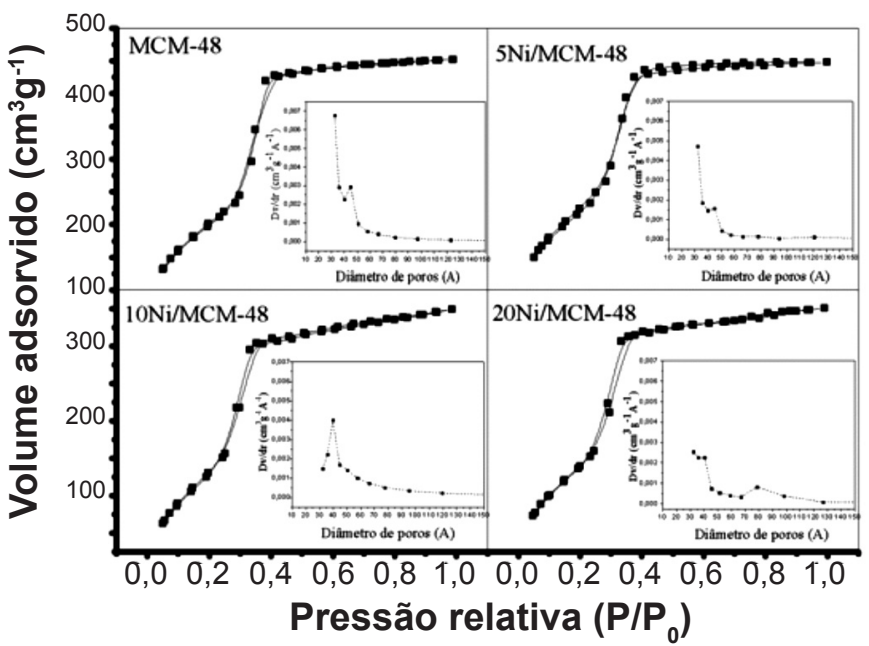

Figura 8: Isotermas de adsorção-dessorção de $\mathrm{N}_{2}$ a $77 \mathrm{~K}$ e distribuição de tamanho de poros das amostras SBA-15, 5Ni/SBA$15,10 \mathrm{Ni} / \mathrm{SBA}-15$ e $20 \mathrm{Ni} / \mathrm{SBA}-15$.

[Figure 8: $N_{2}$ adsorption-desorption isotherms at $77 \mathrm{~K}$ and pore size distribution of the samples $S B A-15,5 \mathrm{Ni} / \mathrm{SBA}-15,10 \mathrm{Ni} / \mathrm{SBA}-15$ and $20 \mathrm{Ni} / \mathrm{SBA}-15$.]

aos sítios de adsorção reduz, progressivamente, os valores de área específica do material [27]. Essa relação não foi observada para as amostras de MCM-48 impregnadas. Isso se deve as diferenças estruturais entre esses mesoporosos. A SBA-15 de estrutura hexagonal altamente ordenada com canais cilíndricos (histerese tipo H2), apresenta estrutura mais favorável ao fenômeno da difusão do que o MCM-48, com sua estrutura cúbica de poros com gargalo estreito e corpo largo (histerese tipo H1).

O diâmetro médio de poros foi estimado através das curvas de distribuição de poros obtidas pelo método BJH e apresentaram valores na faixa de 3,2 a $3,9 \mathrm{~nm}$ para o MCM48 e valores na faixa de 4,0 a $6,7 \mathrm{~nm}$ para o SBA- 15 .

Tabela I - Propriedades texturais das amostras MCM-48, 5Ni/MCM-48, 10Ni/MCM-48, 20Ni/MCM-48, SBA-15, $5 \mathrm{Ni} / \mathrm{SBA}-15,10 \mathrm{Ni} / \mathrm{SBA}-15$ e $20 \mathrm{Ni} / \mathrm{SBA}-15$.

[Table I - Textural properties of samples MCM-48,5Ni/ $M C M-48, \quad 10 \mathrm{Ni} / M C M-48, \quad 20 \mathrm{Ni} / \mathrm{MCM}-48, \quad S B A-15, \quad 5 \mathrm{Ni} /$ SBA-15, 10Ni/SBA-15 and 20Ni/SBA-15.]

\begin{tabular}{cccccc}
\hline Amostra & $\mathrm{a}_{0}(\mathrm{~nm})$ & $\begin{array}{c}\mathrm{Dp} \\
(\mathrm{nm})\end{array}$ & $\begin{array}{c}\mathrm{Wt} \\
(\mathrm{nm})\end{array}$ & $\begin{array}{c}\mathrm{Vp} \\
\left(\mathrm{cm}^{3} \mathrm{~g}^{-1}\right)\end{array}$ & $\begin{array}{c}\mathrm{S}_{\mathrm{BET}} \\
\left(\mathrm{m}^{2} \mathrm{~g}^{-1}\right)\end{array}$ \\
\hline MCM-48 & 8,88 & 3,25 & 1,23 & 0,087 & 924 \\
5Ni/MCM-48 & 8,72 & 3,21 & 1,21 & 0,054 & 904 \\
10Ni/MCM-48 & 8,72 & 3,96 & 0,84 & 0,11 & 971 \\
20Ni/MCM-48 & 8,64 & 3,23 & 1,18 & 0,074 & 798 \\
SBA-15 & 10,49 & 6,78 & 3,71 & 0,56 & 587 \\
5Ni/SBA-15 & 9,95 & 4,02 & 5,93 & 0,57 & 420 \\
10Ni/SBA-15 & 10,95 & 4,03 & 6,92 & 0,64 & 323 \\
20Ni/SBA-15 & 11,51 & 4,51 & 7,00 & 0,48 & 248 \\
\hline
\end{tabular}

$a_{0}=$ Parâmetro de estrutura mesoporosa; $D p=$ Diâmetro de poros; $W t=$ Espessura da parede de sílica; $V p=$ Volume de poros; $S_{B E T}=$ Área especifica. 
As Figs. 9 e 10 mostram as imagens de microscopia eletrônica de varredura das amostras de MCM-48, 5Ni/MCM48, 10Ni/MCM-48, 20Ni/MCM-48 e SBA-15, 5Ni/SBA-15, $10 \mathrm{Ni} / \mathrm{SBA}-15,20 \mathrm{Ni} / \mathrm{SBA}-15$, respectivamente.
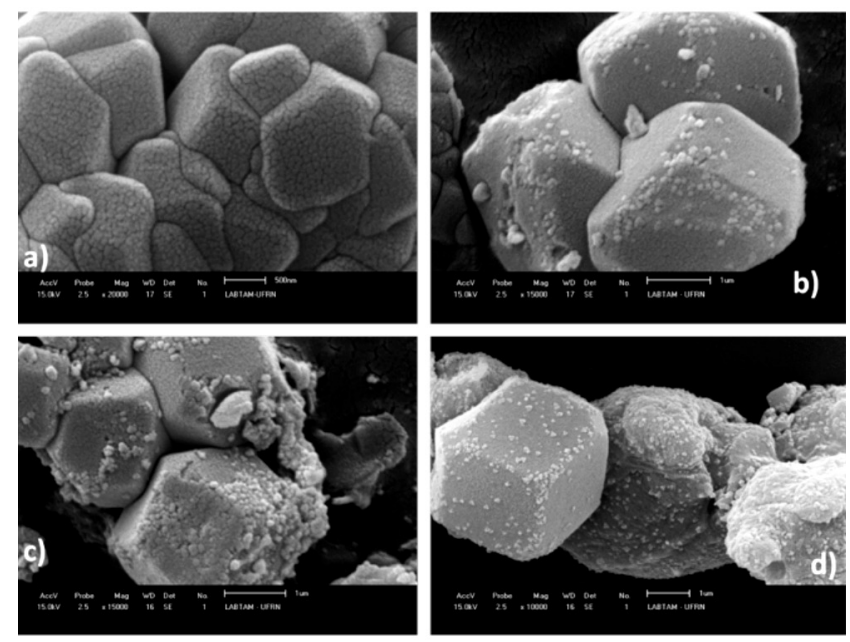

Figura 9: Micrografias obtidas por microscopia eletrônica de varredura das amostras (a) MCM-48, (b) $5 \mathrm{Ni} / \mathrm{MCM}-48$, (c) $10 \mathrm{Ni} /$ MCM-48 e (d) 20 Ni/MCM-48.

[Figure 9: SEM micrographs of samples (a) MCM-48, (b) $5 \mathrm{Ni}$ / $M C M-48$ (c) $10 \mathrm{Ni} / \mathrm{MCM}-48$ and (d) $20 \mathrm{Ni} / \mathrm{MCM}-48$.]

A morfologia das partículas das amostras de MCM-48, 5Ni/MCM-48, 10Ni/MCM-48 e 20Ni/MCM-48 se mostraram similares. Em todos os casos foram observadas partículas na sua maioria uniformes, dando o aspecto de cubos interligados, indicando ser provavelmente essa a fase correspondente ao MCM-48, já que as análises de DRX e adsorção de nitrogênio mostraram que essas amostras são puras e apresentam alto grau de ordenação. Com o aumento de 20000 x, micrografia (a), é possível observar a superfície uniforme e rachaduras oriundas do estado de agregação das partículas. Nas amostras (b) $5 \mathrm{Ni} / \mathrm{MCM}-48$, (c) $10 \mathrm{Ni} /$ MCM-48 e (d) 20Ni/MCM-48, observa-se a presença de rugosidades na superfície dos materiais, tornando-se mais nítidas com o aumento do teor de impregnação. A apresentação de superfícies rugosas nas amostras, observadas através das micrografias, podem ser atribuídas as partículas de $\mathrm{NiO}$ e estão de acordo com os difratogramas de raios $\mathrm{X}$ apresentados, onde a presença de óxido de níquel para os materiais impregnados foi identificada, como também estão de acordo com a distribuição do tamanho de poros, onde foi observada a redução do volume referente aos poros menores e a revelação dos poros maiores do MCM-48, ocasionado pela obstrução dos poros de diâmetros menores pelo $\mathrm{NiO}$ formado na entrada do canal do poro, discutidos anteriormente. Pode-se observar também, para a amostra $10 \mathrm{Ni} / \mathrm{MCM}-48$, que ocorreu uma distribuição bastante heterogênea do óxido de níquel na superfície do material, ocasionando a obstrução de poucos poros em regiões preferenciais da amostra. Como pode ser observada na Tabela I, essa amostra foi a que apresentou maior diâmetro de poro e área específica. Por sua vez, a amostra 20Ni/MCM-48 apresentou uma distribuição bastante homogênea das partículas do óxido de níquel, isso explica o fato dessa amostra apresentar um reduzido tamanho de poro e área específica, mais uma vez devido ao bloqueio da entrada dos canais dos poros. Além disso, de acordo com a literatura [28], a natureza do precursor de níquel influencia na dispersão e no tamanho médio das partículas de óxido de níquel sobre a superfície. Nitrato de níquel como precursor interage fracamente com o suporte de sílica, resultando em cristais grandes na superfície externa do suporte após calcinação. Apenas uma pequena quantidade do componente de metal situa-se no interior dos mesoporos.
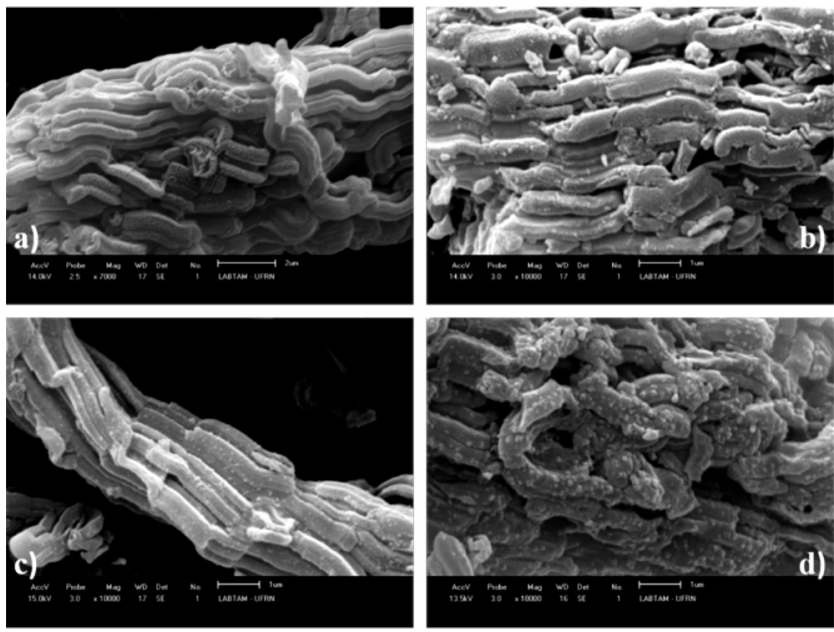

Figura 10: Micrografias obtidas por microscopia eletrônica de varredura das amostras (a) SBA-15, (b) $5 \mathrm{Ni} / \mathrm{SBA}-15$, (c) $10 \mathrm{Ni} /$ SBA-15 e (d) $20 \mathrm{Ni} / \mathrm{SBA}-15$.

[Figure 10: SEM micrographs of samples (a) SBA-15, (b) $5 \mathrm{Ni}$ / SBA-15 (c) 10Ni/SBA-15 and (d) 20Ni/SBA-15.]

As imagens de microscopia eletrônica de varredura (Fig. 10) mostram que as amostras SBA-15, 5Ni/SBA-15, $10 \mathrm{Ni} / \mathrm{SBA}-15$ e $20 \mathrm{Ni} / \mathrm{SBA}-15$ possuem uma morfologia tipo vermicular na forma de tubos cilíndricos, consistindo de agregados tipo corda [12]. Com o aumento de 7000 $\mathrm{x}$, micrografia (a), também é possível observar rachaduras oriundas do estado de agregação das partículas. Nas amostras (b) $5 \mathrm{Ni} / \mathrm{SBA}-15$, (c) $10 \mathrm{Ni} / \mathrm{SBA}-15$ e (d) $20 \mathrm{Ni} / \mathrm{SBA}-$ 15 observa-se a presença de rugosidades na superfície dos materiais, tornando-se mais nítidas com o aumento da concentração de impregnação.Nesse caso, observa-se uma distribuição uniforme do óxido de níquel sobre a superfície e nos poros do material, ao contrário do que ocorreu com o MCM-48. Isso revela o comportamento da distribuição do tamanho de poros e área específica conforme evidenciado pelas análises de DRX e adsorção de nitrogênio (Tabela I).

\section{Testes de adsorção}

A Fig. 11 mostra as isotermas de adsorção de $\mathrm{CO}_{2}$ dos adsorventes investigados.

A adsorção de $\mathrm{CO}_{2}$ apresentou um aumento uniforme ao longo da faixa de pressão investigada. Este fato pode 

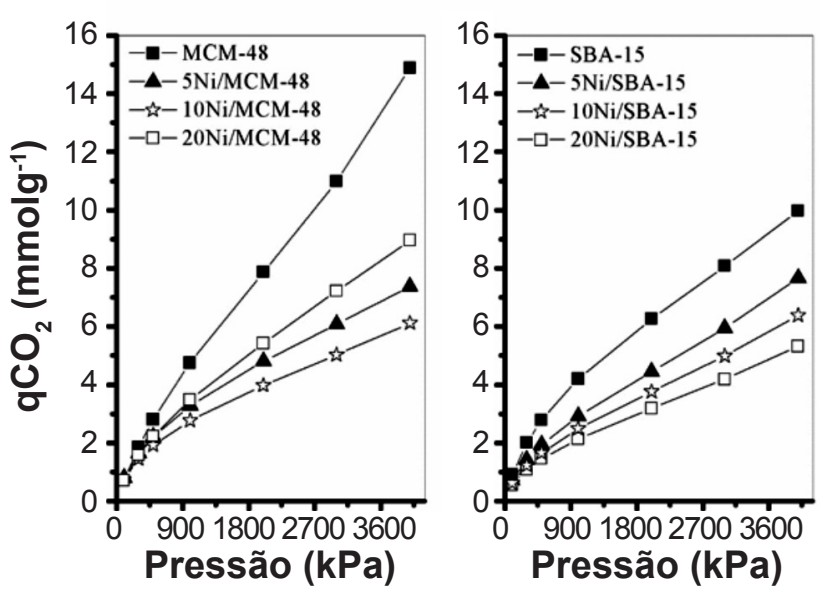

Figura 11: Isotermas de adsorção de $\mathrm{CO}_{2}$ das amostras MCM-48, 5Ni/MCM-48, 10Ni/MCM-48, 20Ni/MCM-48 e SBA-15, 5Ni/SBA-15, 10Ni/SBA-15, 20Ni/SBA-15.

[Figure 11: $\mathrm{CO}_{2}$ adsorption isotherms of the samples MCM48, 5Ni/MCM-48, 10Ni/MCM-48, 20Ni/MCM-48 and $S B A-$ 15, 5Ni/SBA-15, 10Ni/SBA-15, 20Ni/SBA-15.]

ser atribuído ao aumento das interações intermoleculares dentro do reator, onde o quadripolo de dióxido de carbono interage mais fortemente com a superfície a base de sílica dos materiais mesoporosos em pressões mais elevadas, onde há maior aproximação entre adsorbato e os sítios ativos do adsorvente, ocasionando uma maior adsorção de $\mathrm{CO}_{2}[20,29]$.

A modificação desses materiais com diferentes teores de níquel ocasionou uma redução da capacidade de adsorção dos adsorventes, ocorrido possivelmente devido a redução da quantidade de sítios de adsorção proporcionada pela forte interação entre o óxido de níquel e a superfície dos materiais, como também devido ao bloqueio no sistema de poros resultando em limitações difusionais para o $\mathrm{CO}_{2}$. Observa-se uma tendência global em relação à capacidade de adsorção para os materiais investigados da seguinte forma: MCM-48 > 20Ni/MCM-48 > 5Ni/MCM-48 > 10Ni/MCM-48 e SBA-15 > 5Ni/SBA-15 $>10 \mathrm{Ni} / \mathrm{SBA}-15>20 \mathrm{Ni} / \mathrm{SBA}-15$. Para a SBA-15, nota-se uma relação de proporcionalidade entre a diminuição da área específica (Tabela II) e a redução da quantidade de $\mathrm{CO}_{2}$ adsorvido pela amostra quando aumenta-se o teor de óxido de níquel impregnado,possivelmente relacionada ao bloqueio no sistema de poros da SBA-15 devido ao óxido de níquel. Por sua vez, o MCM-48, com estrutura cúbica de poros com gargalo estreito e corpo largo, não apresenta esse comportamento regular quando impregnado. Nesse caso não há relação crescente ou direta de capacidade adsortiva com o teor de níquel. Conforme micrografias apresentadas para as amostras de MCM-48 (Fig. 9), a amostra 20Ni/MCM-48 apresentou uma distribuição bastante homogênea das partículas do óxido de níquel, ocasionando possivelmente um aumento na quantidade de sítios de adsorção,o que proporcionou uma maior capacidade de adsorção de $\mathrm{CO}_{2}$ em comparação as amostras 5Ni/MCM-48 e $10 \mathrm{Ni} / \mathrm{MCM}-48$.

\section{CONCLUSÕES}

Os adsorventes mesoporosos MCM-48 e SBA-15 foram sintetizados e modificados quimicamente por impregnação com óxido de níquel e testados na adsorção de $\mathrm{CO}_{2}$ em uma determinada faixa de pressão. As maiores concentrações de adsorção ocorreram com a pressão em $4000 \mathrm{KPa}$ para os materiais MCM-48 (14,89 mmol g-1) e SBA-15 (9,97 mmol g-1) não impregnados. As capacidades de adsorção seguem a ordem: MCM-48 > 20Ni/MCM-48 > 5Ni/MCM-48 > 10Ni/MCM48 e SBA- $15>5 \mathrm{Ni} / \mathrm{SBA}-15>10 \mathrm{Ni} / \mathrm{SBA}-15>20 \mathrm{Ni} / \mathrm{SBA}-$ 15. Os resultados mostraram que a presença do óxido de níquel nos poros e na superfície dos materiais mesoporosos MCM-48 e SBA-15 resultaram na redução da capacidade de adsorção de $\mathrm{CO}_{2}$ em comparação com os adsorventes puros, indicando que a interação entre o óxido de níquel e a superfície dos materiais, assim como o não bloqueio do sistema de poros tem grande influência neste processo. As diferenças na arquitetura dos canais dos poros e no tipo de estrutura ordenada de cada material foram fatores determinantes das características morfológicas dos sólidos obtidos após impregnação. A estrutura hexagonal com poros cilíndricos presentes na SBA-15 propiciou uma maior uniformidade para o processo de impregnação com nitrato de níquel, com progressiva redução da área específica com o aumento do teor de níquel depositado, conforme evidenciado através das micrografias eletrônicas de varredura e das propriedades texturais para o referido adsorvente. Nesse caso, a adsorção de $\mathrm{CO}_{2}$ foi apenas física, não sofrendo influência da interação entre o óxido de níquel e o gás. Por sua vez, o MCM-48, com estrutura cúbica de poros com gargalo estreito e corpo largo, apresentou comportamento distinto ao que foi observado para a SBA-15, onde o teor de níquel e sua dispersão sobre as partículas do material mesoporoso influenciaram tanto na área específica do sólido como na capacidade de adsorção de $\mathrm{CO}_{2}$. Os resultados obtidos sugerem que os adsorventes mesoporosos investigados apresentam potencial para adsorção de dióxido de carbono em baixas temperaturas e altas pressões.

\section{AGRADECIMENTOS}

Ao LCR/NUPPRAR (Laboratório de Catálise e Refine de Processamento Primário e Reuso de Água Produzida e Resíduos) da Universidade Federal do Rio Grande do Norte e à CAPES (Coordenação de Aperfeiçoamento de Pessoal de Nível Superior) pelo apoio financeiro.

\section{REFERÊNCIAS}

[1] International Energy Agency, "The Technology Roadmap, Carbon capture and storage", France (2013).

[2] S. M. Benson, F. M. Orr Jr, Carbon dioxide capture and storage, Mater. Res. Soc. Bull. 33 (2008) 303-305.

[3] A. Macario, A. Katovic, G. Giordino, F. Iucolano, D. Caputo, Synthesis of mesoporous materials for carbon dioxide sequestration, Microporous Mesoporous Mater. 81 
(2005) 139-147.

[4] T. Nguyen, M. Hilliard, G. T. Rochelle, Amine volatility in $\mathrm{CO}_{2}$ capture, Int. J. Greenhouse Gas Control 4 (2010) 707-715.

[5] M. R. Mello, D. Phanon, G. Q. Silveira, N. Llewelly, C. M. Ronconi, Amine-modified MCM-41 mesoporous silica for carbon dioxide capture, Microporous Mesoporous Mater. 143 (2011) 174-179.

[6] J. L. Figueiredo, F. R. Ribeiro, "Catálise Heterogênea", Fundação Calouste Gulbenkian, Lisboa, Portugal (1989).

[7] R. Ciola, "Fundamentos da Catálise", Ed. Univ. S. Paulo, Editora Moderna, S. Paulo, SP (1981).

[8] M. Bhagiyalakshmi, P. Hemalatha, M. Ganesh, M. M. Peng, H. T. Jang, Synthesis of copper exchanged heteropolyacids supported on MCM-48 and its application for $\mathrm{CO}_{2}$ adsorption, J. Ind. Eng. Chem.17 (2011) 628-632.

[9] A. Zukal, C. O. Arean, M. R. Delgado, P. Nachtigall, A. Pulido, J. Mayerová, J.Cejka, Combined volumetric, infrared spectroscopic and theoretical investigation of $\mathrm{CO}_{2}$ adsorption on Na-A zeólita, Microporous Mesoporous Mater.146 (2011) 97-105.

[10] C. T. Kresge, M. E. Leonowicz, W. J. Roth, J. C. Vartuli, J. S. Becck, Ordered mesoporous molecular sieves synthesized by a liquid-crystal template mechanism, Nature 359 (1992) 710-712.

[11] P. Selvam, S. E. Dapurkar, Catalytic activity of highly ordered mesoporous VMCM-48, Appl. Catalysis A: General 276 (2004) 257-265.

[12] D. Zhao, J. Feng, Q. Huo, N. Melosh, G. H. Fredrickson, B. F . Chmelka, G. D. Stucky, Science 279 (1998) 548.

[13] J. Shi-lin, Z. Hua, L. Zhang, J. Mater. Chem. 14 (2004) 795-806.

[14] G. E. Luz Jr, A. C. R. Melo, S. H. Lima, A. S. Araujo, V. Fernandes Jr, Desidratação de etanol sobre material nanoestruturado do tipo La-SBA-15, Quimica Nova 33, 8 (2010) 1646-1648.

[15] D. Jang, S. Park, Influence of nickel oxide on carbon dioxide adsorption behaviors of activated carbons, Fuel 102 (2012) 439-444.

[16] T. G. Oliveira, S. W. M. Machado, S. C. G. Santos, M. J. B. Souza, A. M. G. Pedrosa, Adsorção de $\mathrm{CO}_{2}$ em peneiras moleculares micro e mesoporosas, Quim. Nova 37, 4 (2014) 610-617.

[17] F. Meshkani, M. Rezaei, M. Andache, Investigation of the catalytic performance of $\mathrm{Ni} / \mathrm{MgO}$ catalysts in partial oxidation, dry reforming and combined reforming of methane, J. Ind. Eng. Chem. 20 (2014) 1251-1260.
[18] R. Wojcieszak, S. Monteverdi, M. Mercy, I. Nowak , M. Ziolek, M. M. Bettahar, Nickel containing MCM-41 and AlMCM-41 mesoporous molecular sieves characteristics and activity in the hydrogenation of benzene, Appl. Catalysis A: General 268 (2014) 241-253.

[19] M. Laribi, K. Bachari, R. Chebout, M. Touati, Synthesis, characterization and catalytic application of the nickel containing mesoporous silica, J. Assoc. Arab. Univ. Basic Appl. Sci. 12 (2012) 42-47.

[20] A. S. Frey, O. Hinrichsen, Comparison of differently synthesized $\mathrm{Ni}(\mathrm{Al}) \mathrm{MCM}-48$ catalysts in the ethane to propene reaction, Microporous Mesoporous Mater. 164 (2012) 164-171.

[21] S. Park, S. Lee, A study on hydrogen-storage behaviors of nickel-loaded mesoporous MCM-41, J. Colloid Interface Sci. 346 (2010) 194-198.

[22] A. Doyle, B. K. Hodnett, Stability of MCM-48 in aqueous solution as a function of $\mathrm{pH}$, Microporous Mesoporous Mater. 63 (2003) 53-57.

[23] S. Brunauer, P. H. Emmett, E. Teller, Adsorption of gases in multimolecular layers, J. Am. Chem. Soc. 60 (1938) 309. [24] S. Brunauer, "The Adsorption of Gases and Vapors", University Press, Oxford, UK (1945).

[25] D. Liu, X. Y.Quek, W. N. E. Cheo, R. Lau, A. Borgna, Y. Yang, MCM-41 supported nickel-based bimetallic catalysts with superior stability during carbon dioxide reforming of methane: Effect of strong metal-support interaction, J. Catalysis 266 (2009) 380-390.

[26] M. Kruk, M. Jaroniec, H. J. Shin, R. Ryoo, S. H. Joo, Optimization of silica/surfactant ratio in MCM-41 synthesis, Studies Surfaces Sci. Catalysis 156 (2005) 55-62.

[27] J. S. Beck, J. C. Vartuli, W. J. Roth, M. E. Leonowicz, C. T. Kresge, K. D. Schmitt, C. T. Chu, D. H. Olson, E. W. Sheppard, S. B. Mccullen, J. B. Higgins, J. L. Schlenker, A new family of mesoporous molecular sieves prepared with liquid crystal templates, J. Am. Chem. Soc. 114 (1992) 10834-10843.

[28] M. K. Montañez, R. Molina, S. Moreno, Nickel catalysts obtained from hydrotalcites by coprecipitation and urea hydrolysis for hydrogen Production, Int. J. Hydrogen Energy 39 (2014) 8225-8237.

[29] X. Wang, X. Ma, C. Song, D. R. Locke, S. Siefert, R. E. Winans, J. Möllmer, M. Lange, A. Möller, R. Gläser, Molecular basket sorbents polyethylenimine-SBA-15 for $\mathrm{CO}_{2}$ capture from flue gas: Characterization and sorption properties, Microporous Mesoporous Mater. 169 (2013) 103-111.

(Rec.25/06/2014, Rev.07/07/2014, Ac. 10/07/2014) 